\title{
Metachronous Mesorectal Recurrence after Colectomy for Ascending Colon Cancer
}

\author{
Matthew Mackowsky ${ }^{a} \quad$ Ashley Toussaint ${ }^{a} \quad$ Kevin Clarke $^{b}$ \\ Rachel Hudacko ${ }^{c}$
}

aDepartment of Surgery, Monmouth Medical Center, Long Branch, NJ, USA; bepartment of Surgery, Newark Beth Israel Medical Center, Newark, NJ, USA; 'Department of Pathology and Laboratory Medicine, Rutgers Robert Wood Johnson Medical School, New Brunswick, NJ, USA

\section{Keywords}

Adenocarcinoma $\cdot$ Advanced colorectal carcinoma $\cdot$ Cancer $\cdot$ Carcinoma $\cdot$ Familial adenomatous polyposis - Mesorectal recurrence $\cdot$ Mesorectum $\cdot$ Metachronous recurrence . Recurrence

\section{Abstract}

Background: An isolated metachronous recurrence in the mesorectum from a primary ascending colon cancer is a rare finding that has not been previously reported. This may represent a form of retroperitoneal spread, sometimes referred to as "drop metastasis," which is an uncommon mechanism for metachronous recurrence. Case Presentation: A 38-year-old male presented to the Emergency Department in January of 2018 with profound anemia. A colonoscopy revealed innumerable colonic polyps. He reported having multiple family members diagnosed with colon cancer and was subsequently diagnosed with familial adenomatous polyposis with rectal sparing. Total abdominal colectomy with ileorectal anastomosis was performed, revealing a T3N1a adenocarcinoma of the ascending colon. The patient subsequently underwent 12 cycles of adjuvant FOLFOX. Surveillance imaging in late 2019 revealed a suspicious mass in the superior perirectal soft tissue without any other sites of potential disease. Completion proctectomy was performed in January 2020, 2 years after the initial resection. Pathology revealed a mesorectal tumor deposit located $1.5 \mathrm{~cm}$ distal to the ileorectal anastomosis. No evidence of mucosal involvement or nodal metastasis was identified. Conclusion: Isolated mesorectal recurrence is a rare and previously unreported clinical finding following resection of an ascending colon cancer with an ileorectal anastomosis. This likely represents a form of retroperitoneal spread. 
Mackowsky et al.: Mesorectal Adenocarcinoma Recurrence following Colectomy

\section{Introduction}

Colon cancer is currently the third most commonly diagnosed cancer in both men and women in the USA, and is also the third leading cause of cancer-related deaths [1]. Of patients with colon cancer, approximately $20 \%$ present with synchronous metastatic disease [1]. For those patients who undergo resection for colon cancer, $40 \%$ experience disease recurrence [2]. Isolated locoregional disease is the initial site of recurrence in $10-20 \%$ of patients [2]. Colon cancer progresses via local invasion as well as both hematogenous and lymphatic metastasis. The most frequent sites of distant colon cancer metastasis include the liver, lungs, and peritoneum. Many unusual metastatic sites have been documented in the literature. These sites included the pancreas, uterus, penis, scrotum, prostate, bladder, and bones [3].

Two of the most common familial colon cancer syndromes are familial adenomatous polyposis (FAP) and Lynch syndrome (together accounting for approx. 5\% of cases of colorectal cancer, with FAP accounting for $<1 \%$ ). FAP is characterized by a germline mutation in the adenomatous polyposis coli (APC) gene on chromosome 5 which causes numerous colonic adenomas $(>100)$ during adolescence. This mutation has an autosomal dominant inheritance with near-complete penetrance; however, $25 \%$ of cases are due to de novo mutations. Colectomy is recommended for patients with FAP and a high polyp number count due to unrealistic endoscopic control. Surgical options for FAP include proctocolectomy with end ileostomy, total proctocolectomy with ileal pouch anal anastomosis, or total abdominal colectomy with ileorectal anastomosis for FAP with rectal sparing [4].

This case report discusses a case of metachronous recurrence from an ascending colon cancer with mesorectal tumor deposition in a patient with FAP. To the best of the authors' knowledge, there exists only one other report of an isolated mesorectal recurrence following colectomy for colon cancer [5].

\section{Case Presentation}

The patient, a 38-year-old male previously undiagnosed with FAP despite a strong family history, initially presented to the surgical service following a syncopal episode in December of 2017. Workup revealed severe anemia and a hemoglobin level of $3.8 \mathrm{~g} / \mathrm{dL}$ (reference range: 12-16). Colonoscopy revealed innumerable colonic polyps beginning at the cecum and extending to the sigmoid colon, with sparing of the rectum, and the patient was diagnosed with FAP. A total abdominal colectomy with ileorectal anastomosis was performed without complication (Fig. 1). Pathology subsequently revealed a T3 adenocarcinoma of the ascending colon with 1 positive lymph node out of 75 examined. The patient subsequently received 12 cycles of adjuvant FOLFOX.

Surveillance CT scanning in late 2019 revealed a suspicious mass in the superior perirectal soft tissue (Fig. 2). The patient exhibited no related symptoms at that time. Follow-up PET scanning revealed a PET-avid mass within the left superior mesorectal soft tissue, corresponding to the mass noted on the CT scan (Fig. 2). No other suspicious lesions were identified. An examination under anesthesia was conducted, revealing an extrinsic nodular mass in the area of the left superior rectal wall, approximately $2 \mathrm{~cm}$ below the level of the ileorectal anastomosis. Colonoscopy and biopsy revealed 2 tubular adenomas, but no mucosal mass corresponding to the lesion noted on imaging. The patient underwent completion proctectomy with an ileoanal anastomosis and diverting loop ileostomy in January of 2020. The patient's postoperative course was uneventful, and he was discharged without complication. Pathology revealed a metastatic tumor deposit within the mesorectum $1.5 \mathrm{~cm}$ distal to the ileorectal anastomosis, with no evidence of mucosal or nodal involvement (Fig. 3). The lesion 


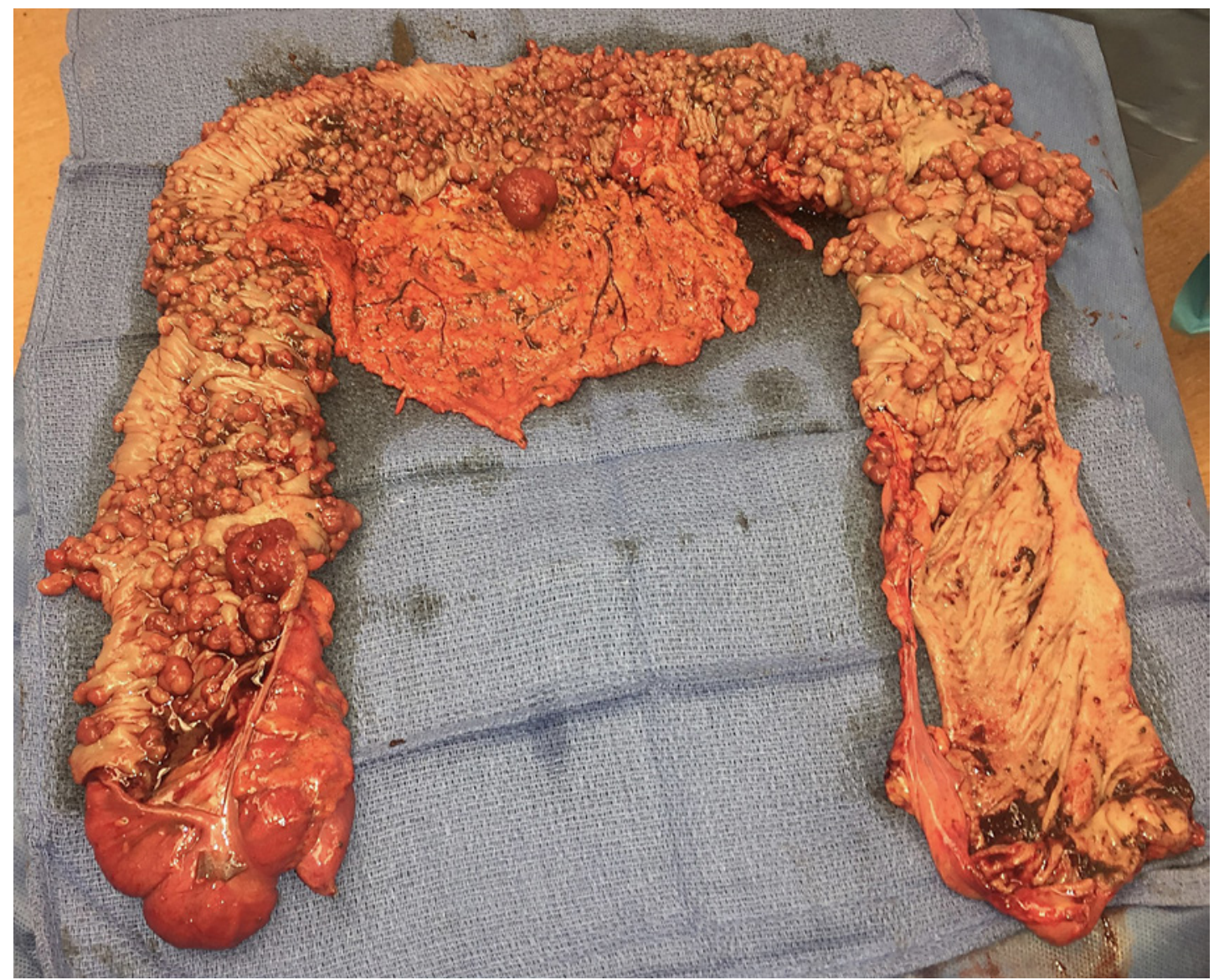

Fig. 1. Gross specimen from index operation (abdominal colectomy with ileorectal anastomosis). Polyposis is consistent with familial adenomatous polyposis with sparing of the rectum and distal sigmoid.

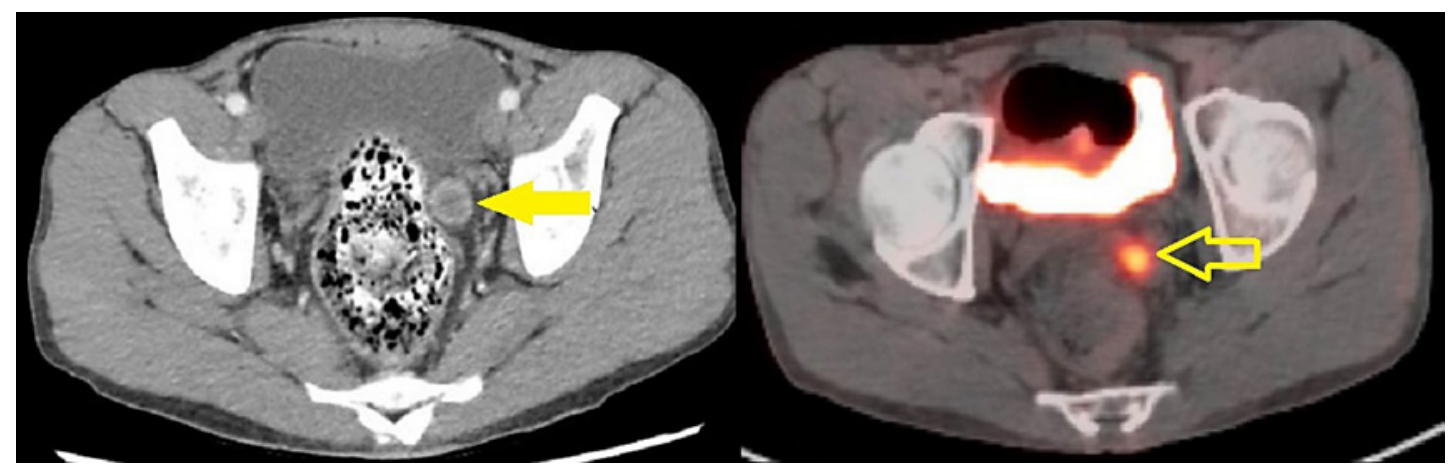

Fig. 2. CT (left) and PET (right) images from preoperative surveillance imaging. The filled arrow denotes mesorectal occurrence with the plane separating the rectal wall from the site of recurrence on the CT scan. The hollow arrow denotes the site of hypermetabolic activity on the PET scan.

had been resected in its entirety. There was no evidence of a primary rectal cancer, nor was there evidence of involvement of the ileal mesentery. After discussion at a multidisciplinary tumor board, the patient was subsequently returned to surveillance without further plans for adjuvant therapy. 
Fig. 3. Histologic section of the perirectal mass showing an adenocarcinoma with irregular borders infiltrating the fibroadipose tissue. No evidence of a preexisting lymph node or vessel was noted in this tumor deposit. HE. $\times 40$.

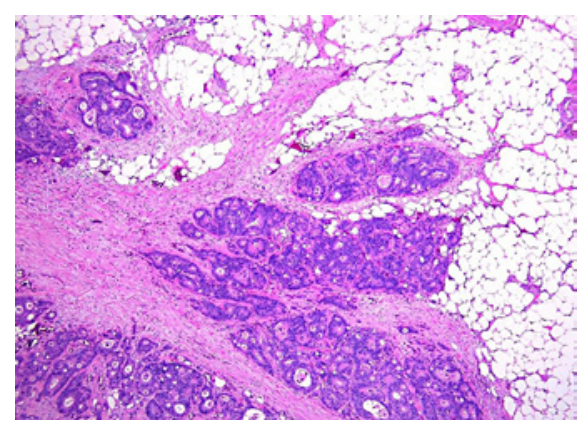

\section{Discussion}

Metachronous tumor deposition in the mesorectum following colectomy is rare. Only one additional case report has been noted in the literature [5]. Kalaitzis et al. [5] reported a case of metachronous spread to the mesorectum as well as the abdominal wall 1 year following resection of a sigmoid colon cancer. The patient had been intended for resection, but ultimately underwent chemoradiation after cancellation of resection following an intraoperative myocardial infarction.

In the current case, a 38-year-old male with FAP and rectal sparing presented with a new perirectal mass 2 years following subtotal colectomy for an ascending colon cancer with adequate resection margins and adequate lymph node harvest. The mass appeared resectable, and the patient underwent a completion proctectomy and ileoanal anastomosis. Pathology revealed a tumor deposit within the perirectal soft tissue, without mucosal involvement, nodal metastasis, or evidence of a new rectal cancer.

The pattern of recurrence in this case is unique. While perianastomotic recurrences are not uncommon, this particular recurrence was a non-mucosa-based tumor deposit within the mesorectal soft tissue, $1.5 \mathrm{~cm}$ distal to the level of the anastomosis. The mesorectum is a hindgut derivative with blood supply originating from the superior rectal, middle rectal, and inferior rectal vessels (terminal branches from the inferior mesenteric artery, and branches from the internal iliac and internal pudendal arteries, respectively). Venous outflow from the ascending colon and rectum does not intersect until the confluence of the superior mesenteric and the splenic vein. In addition, antegrade lymphatic flow from these sources does not intersect until the level of the preaortic lymph nodes.

A variety of mechanisms have been postulated as being responsible for locally recurrent colorectal cancers. The most common model for local recurrence divides locoregional recurrence into four categories: (1) true perianastomotic recurrence via mural spread; (2) mesenteric spread via lymphatics; (3) peritoneal metastasis; and (4) retroperitoneal spread. So-called drop metastases via a retroperitoneal pathway are among the least common forms of locoregional recurrence $[2,6]$. In the current instance of an ascending colon cancer with metachronous spread to the mesorectum, retroperitoneal spread seems to be the most likely source. Given the distance from the mesentery of the ascending colon and the mesorectum, as well as the lack of a mucosal source, a true perianastomotic (via mural extension), mesenteric source (via regional nodal extension), or peritoneal disease are far less likely mechanisms for a mesorectal recurrence at an ileorectal anastomosis [2]. As reported in a Memorial Sloan Kettering Cancer Center series of 100 patients suffering locoregional recurrence following colonic resection, retroperitoneal spread of disease is the least frequent form of locoregional recurrence [6]. Colocolonic and colorectal anastomotic recurrence are not uncommon [7]; however, recurrence at the mesorectum distal to the site of an ileorectal anas- 
tomosis has not been reported. The pattern of the recurrence in this case likely represents a form of "drop metastasis." To the authors' knowledge, this is the first instance of this type of recurrence being reported at an ileorectal anastomosis.

A survival benefit has been noted for appropriately selected patients with metachronous recurrences who are able to undergo R0 resection [8]. The National Comprehensive Cancer Network (NCCN) guidelines currently suggest resection of metachronous metastasis when possible [9]. The NCCN guidelines do not differentiate between sites of metachronous recurrence. For the subject of this paper, after discussion at a multidisciplinary tumor board, given the lack of evidence of distant metastatic spread, and the resectability of the recurrence, the patient underwent resection. The NCCN guidelines also suggest no adjuvant therapy following resection of a locoregional recurrence for those who have already had oxaliplatin-based chemotherapy. The authors followed this suggestion and the patient was returned to surveillance.

\section{Conclusion}

Isolated mesorectal recurrence is a rare clinical finding following resection of a primary ascending colon cancer. This article presents a case of a metachronous mesorectal recurrence in a patient with FAP who had undergone total abdominal colectomy approximately 2 years previously. The patient subsequently underwent completion proctectomy with ileoanal anastomosis. Pathology revealed a metastatic tumor deposit in the mesorectum. This is the first instance reported of an isolated mesorectal recurrence following resection of an ascending colon cancer with an ileorectal anastomosis. This likely represents a form of retroperitoneal spread.

\section{Statement of Ethics}

The subject gave written informed consent to publish his case (including publication of images).

\section{Disclosure Statement}

The authors have no conflicts of interest to disclose.

\section{Funding Sources}

There were no funding sources.

\section{Author Contributions}

All authors are major contributors to the publication. 


\section{References}

1 Siegel R, Miller K, Jemal A. Cancer statistics, 2020. CA Cancer J Clin. 2020;70(1):7-30.

2 Landmann RG, Weiser MR. Surgical management of locally advanced and locally recurrent colon cancer. Clin Colon Rectal Surg. 2005;18(3):182-9.

3 Sánchez L, Dellamea M, Moreno L, Osorio C, González Toranzo R, Sosa M. Unexpected destinies: unusual sites of colorectal cancer metastases. Imagenes. 2015;4(12):37-45.

4 Bisgaard ML, Fenger K, Bülow S, Niebuhr E, Mohr J. Familial adenomatous polyposis (FAP): frequency, penetrance, and mutation rate. Hum Mutat. 1994;3(2):121-5.

5 Kalaitzis J, Filippou G, Zizi-Sermpetzoglou A, Marinis A, Hadjimarcou A, Paschalidis N, et al. Case of a sigmoid colon cancer with metachronous metastases to the mesorectum and the abdominal wall. World J Surg Oncol. 2010;8:17.

6 Bowne WB, Lee B, Wong WD, Ben-Porat L, Shia J, Cohen AM, et al. Operative salvage for locoregional recurrent colon cancer after curative resection: an analysis of 100 cases. Dis Colon Rectum. 2005;48(5):897-909.

7 Funahashi K, Koike J, Saito N, Shiokawa H, Shirasaka K, Teramoto T. A rare case of repeated anastomotic recurrence due to tumor implantation after curative surgery for sigmoid colon cancer. World J Surg Oncol. 2007; 5:91.

8 Chesney TR, Nadler A, Acuna SA, Swallow CJ. Outcomes of resection for locoregionally recurrent colon cancer: a systematic review. Surgery. 2016;160(1):54-66.

9 National Comprehensive Cancer Network. Colon [accessed 2020 Feb 9]. Available from: https://www.nccn. org/professionals/physician_gls/pdf/colon.pdf. 